\title{
Prevalência e fatores associados ao tabagismo em uma unidade universitária
}

\author{
Jéssica Steffany Miranda*, Janaina Benatti de Almeida*, Silvio Fernando Guideti Marques**
}

*Acadêmicas do Curso de Enfermagem do Centro Universitário de Lins (UNILINS), Lins/SP,

*** Docente do Curso de Enfermagem do Centro Universitário de Lins (UNILINS)

\section{Resumo}

O tabagismo constitui um sério problema de saúde pública. Este estudo teve como objetivo conhecer a prevalência do tabagismo e descrever o perfil e hábitos do consumo tabágico entre os alunos do Centro Universitário Católico Salesiano Auxilium de Lins/SP, e servir como medida auxiliar para o desenvolvimento de estratégias que visem à profilaxia do consumo de cigarros nesta população. Um total de 182 alunos respondeu a um questionário com perguntas dirigidas a fumantes, ex-fumantes e não fumantes. A prevalência de tabagismo foi de $11,0 \%$. 84,6\% dos alunos não eram fumantes e 4,4\% foram classificados como ex-fumantes. Da amostra total, 123 eram mulheres (67,6\%). A média da idade da população estudada foi de 22,8 $\pm 3,6$ anos. Os cursos de Química (40,0\%), Terapia Ocupacional (40,0\%), História (20,0\%), Psicologia (18,2\%) e Enfermagem $(17,6 \%)$ apresentaram as maiores prevalências de fumantes. A idade média de início no tabagismo foi de 17,8 $\pm 7,4$ anos. Foi observado consumo médio de $8,3 \pm 7,8$ cigarros por dia. Todos os universitários que se declararam fumantes utilizam cigarro industrializado. Este índice de prevalência assemelha-se aos de outros Centros Universitários e demanda políticas de controle do tabagismo nos meios universitários.

Palavras-chave: tabagismo, universitários, prevalência.

\section{Abstract \\ Prevalence and characteristics of smoking in the university unit}

The smoking is a serious public health problem. This study aims to determine the prevalence of nicotine addiction and understand the profile and habits of the addicted students of Catholic University Centre Salesiano Auxilium of Lins/SP, and serve as auxiliary measure for the development of strategies aimed at prevention of smoking in this population. A total of 182 students responded to a smoking questionnaire, including questions directed at smokers, nonsmokers and former smokers. The prevalence of nicotine addiction was $11.0 \%$. About $84.6 \%$ of the students were not smokers and $4.4 \%$ were classified as former smokers. In the study sample, 123 of the students were women (67.6\%). The average age of the population was 22.8 \pm 3.6 years. The percentage of smokers was higher in the Chemistry, Occupational Therapy, History, Psychology and Nursing $(40.0 \%, 40.0 \%, 20.0 \%, 18.2 \%$ and $17.6 \%$, respectively). The average age of start in the smoking was of $17.8 \pm 7.4$ years and average consumption was $8.3 \pm 7.8$ cigarettes per day. All of the consumers used industrialized cigarettes. This prevalence correlates to the other academic centers and demands politics of nicotine addiction control among the university centers.

Key-words: smoking, university students, prevalence. 


\section{Resumen}

\section{La prevalencia y factores asociados al tabaquismo en una unidad universitaria}

El tabaquismo es un grave problema de salud pública. Este estudio tiene como objetivo conocer la prevalencia del tabaquismo y comprender el perfil y hábitos de consumo entre los estudiantes universitarios del Centro Universitario Católico Salesiano Auxilium de Lins/SP, y servir como medida auxiliar para el desarrollo de estrategias dirigidas a la prevención del consumo de tabaco en esta población. Un total de 182 estudiantes contestaron a un cuestionario con preguntas a fumadores, no fumadores y ex fumadores. La prevalencia de tabaquismo fue de un 11\%. 84,6\% de los estudiantes no eran fumadores y el $4,4 \%$ ex fumadores. De la muestra total, 123 eran mujeres $(67,6 \%)$. La edad media de la población estudiada fue de $22,8 \pm$ 3,6. El porcentaje de fumadores más alto se dio en las carreras de Química (40,0\%), Terapia Ocupacional (40,0\%), Historia (20,0\%), Psicología (18,2\%) y Enfermería (17,6\%). La edad media de inicio en el consumo de tabaco fue de 17,8 $\pm 7,4$ y el consumo medio 8,3 \pm 7,8 cigarrillos industrializados al día. Este índice de prevalencia se asemeja a otros centros universitarios y demanda políticas de control del tabaquismo en los recintos universitarios.

Palabras-clave: tabaquismo, estudiantes universitarios, predominio, característica.

\section{Introdução}

O tabagismo é responsável por altos índices de morbimortalidade mundial, sendo considerado pela Organização Mundial da Saúde (OMS) a principal causa de morte evitável em todo o mundo [1].

Levantamento realizado em 2005 apontou $10,1 \%$ da populaçáo brasileira de 12 a 65 anos como sendo dependente de tabaco. A expectativa de vida de um fumante é $25 \%$ menor que a de um não fumante [2]. Se não forem tomadas medidas adequadas para o controle dessa pandemia, próximo ao ano de 2020, o tabagismo será responsável por 10 milhóes de mortes por ano, com proporção de uma em cada seis pessoas consumidoras de tabaco mundialmente. Desses óbitos, 7 milhóes ocorrerão nos países em desenvolvimento [3].

Diversos estudos realizados em nosso país $[4,5]$ e no mundo [6,7] apontam que o hábito de fumar se instala precocemente, já que $80 \%$ dos atuais adultos fumantes declararam ter iniciado nesta prática antes dos dezoito anos de idade. Ressalta-se, também, que a idade de experimentação e início do tabagismo precoce, ou seja, antes dos 20 anos de idade, está comumente correlacionada ao período de transição do indivíduo do nível de ensino médio para o superior [8].

Estas informaçóes confirmam a tendência mundial de aumento da prevalência do uso de cigarros entre a população de adolescentes e adultos jovens, principalmente entre os estudantes universitários, jovens estes considerados público com grande suscetibilidade de envolvimento com o tabaco [8]. Diante da gravidade desse quadro, vários estudos sugerem que medidas antitabágicas sejam direcionadas prioritariamente a esta populaçáo $[9,10]$.
Portanto, este trabalho teve como objetivo conhecer a prevalência e descrever o perfil e hábitos do consumo tabágico entre os estudantes do Centro Universitário Católico Salesiano Auxilium de Lins (Unisalesiano-Lins/SP), e servir como medida auxiliar para o desenvolvimento de estratégias que visem à profilaxia do consumo de cigarros nesta população.

\section{Materiais e métodos}

Realizou-se um estudo transversal com os alunos do Unisalesiano-Lins/SP, devidamente matriculados nos cursos de graduação durante o segundo semestre de 2008. Para se calcular o tamanho da amostra, utilizou-se a população total de 2185 universitários matriculados e erro amostral tolerável de $5 \%$ para possibilitar intervalo de confiança de 95\%. Dessa maneira, foi obtida, inicialmente, como amostra ideal para o desenvolvimento deste estudo, um grupo de 182 universitários [11].

Para a obtenção da amostra foi realizado sorteio aleatório simples entre os alunos de todos os cursos oferecidos pelo Unisalesiano-Lins/SP. O número de alunos entrevistados por curso foi determinado através do produto entre o fator de proporcionalidade, que relaciona o tamanho da amostra com o tamanho da população estudada, e o número total de alunos do respectivo curso.

Para se obterem as informações necessárias sobre o consumo de tabaco, utilizou-se um questionário pré-codificado, individual e confidencial, adaptado da Organização Mundial da Saúde, validado no Brasil pelo Instituto Nacional do Câncer, com perguntas relacionadas ao hábito tabágico, distribuídas em três domínios, direcionadas aos fumantes regulares e fumantes ocasionais, aos ex-fumantes 
e aos não fumantes. Este questionário continha variáveis demográficas (idade, sexo e cor), socioeconômicas (renda familiar em salários mínimos vigentes na época da pesquisa), variáveis relacionadas ao tabagismo (se é fumante, tipo de fumo, consumo diário, idade de início, tempo de tabagismo e, caso ex-fumante, tempo de tabagismo e de suspensão do fumo), tipo de tabaco (industrializado, confeccionado pelo próprio fumante, charuto e cachimbo), convivência com fumantes (pais, irmãos, amigos) e local de convivência (casa, universidade, trabalho). Também foram investigados o consumo de bebidas alcoólicas, prática de esportes e presença de sintomas como tosse seca, produtiva e "chiado no peito".

O ponto de corte para a definiçáo de fumante foi ter fumado um ou mais cigarros por dia há pelo menos um mês, e para ex-fumantes aqueles que na ocasiáo da pesquisa náo eram fumantes, mas que o haviam sido no passado.

O questionário foi aplicado diretamente aos alunos após esclarecimentos e orientaçóes básicas sobre os objetivos da pesquisa em questáo, ficando o universitário livre para decidir sobre sua participação.

Esta pesquisa teve aprovação do Comitê de Ética em Pesquisa (protocolo 01/2008) do Centro Universitário de Lins (Unilins) e atende à resoluçáo no. 196, de outubro de 1996, do Conselho Nacional de Saúde do Ministério da Saúde (CNS). Nele estão garantidos os consentimentos livres e esclarecidos, a privacidade, a confidencialidade e o anonimato, com respeito aos valores socioculturais.

Foi utilizado o teste do qui-quadrado para avaliar a existência de associaçóes entre as variáveis analisadas, sendo considerado o valor de $\mathrm{p}<0,05$ para significância estatística.

\section{Resultados}

Foram entrevistados 182 alunos, dos quais 154 se declararam náo fumante, representando $84,6 \% \mathrm{da}$ amostra. A prevalência de tabagismo foi de $11,0 \%$ (20), sendo 75,0\% de fumantes regulares (15) e 25,0 $\%$ de fumantes ocasionais (5). Como ex-fumantes foram classificados 4,4\% dos alunos (8).

Em relaçáo à distribuiçáo da amostra de acordo com o sexo, foi encontrada diferença estatisticamente significativa $(\mathrm{p}<0,05)$, uma vez que $67,6 \%$ dos entrevistados eram do sexo feminino, e dentre os universitários tabagistas, $80,0 \%$ pertenciam a este gênero.
A média de idade da população estudada foi de 22,8 \pm 3,6 anos. Em relação à idade de início do tabagismo, pôde-se observar que $65 \%$ dos entrevistados fumantes começaram a fumar com idade entre 15 e 19 anos (13) (Tabela I). A média declarada de idade em que se iniciou o hábito tabágico foi de $16,9 \pm 2,9$ anos.

Tabela I - Características do tabagismo entre estudantes do Unisalesiano-Lins/SP quanto à idade de início do tabagismo, número de cigarros fumados por dia e tempo de fumo no ano de 2009.

\begin{tabular}{lll}
\hline & $\mathrm{n}$ & $\%$ \\
\hline Idade de início & & \\
10 a 14 anos & 3 & 15,0 \\
15 a 19 anos & 13 & 65,0 \\
20 a 24 anos & 4 & 20,0 \\
$>25$ anos & 0 & 0,0 \\
\hline Número de cigarros por dia & & \\
\hline 1 a 5 cigarros/dia & 8 & 40,0 \\
6 a 10 cigarros/dia & 8 & 40,0 \\
11 a 15 cigarros/dia & 0 & 0,0 \\
16 a 20 cigarros/dia & 2 & 10,0 \\
$>20$ cigarros/dia & 2 & 10,0 \\
\hline Tempo de fumo & & \\
\hline$<1$ ano & 2 & 10,0 \\
1 a 3 anos & 3 & 15,0 \\
4 a 6 anos & 10 & 50,0 \\
$>6$ anos & 5 & 25,0 \\
\hline
\end{tabular}

Considerando os fumantes regulares e ocasionais como um único grupo de fumantes, verificou-se que os cursos com as maiores prevalências de tabagismo foram: Química (40,0\%), Terapia Ocupacional (40,0\%), História (20,0\%), Psicologia (18,2\%), Enfermagem (17,6\%) e Letras (14,3\%) (Figura 1). Já os cursos de Matemática, Administração, Ciências Contábeis e Pedagogia náo apresentaram nenhum dos estudantes entrevistados como fumantes.

A renda familiar acima de 4,0 a 7,0 salários mínimos foi predominante $(70 \%)$ no grupo de tabagistas (Tabela II).

Tabela II - Renda familiar em salários mínimos no grupo tabagista.

\begin{tabular}{lll}
\hline Renda Familiar (salários mínimos) & $\mathbf{n}$ & $\%$ \\
\hline$<1,5$ & 0 & 0,0 \\
1,5 a 3,9 & 5 & 25,0 \\
4,0 a 7,0 & 14 & 70,0 \\
$>7,0$ & 1 & 5,0 \\
\hline
\end{tabular}


Em relação à questão sobre os tipos de tabaco utilizados pelos universitários, foi observado que todos os entrevistados (20) utilizavam cigarros industrializados. Em média, fumavam-se 8,4 \pm 7,9 cigarros por dia e $80,0 \%$ (16) dos alunos consumiam entre um e cinco cigarros por dia (Tabela I).

Ao se avaliar a distribuição conjunta do número de anos em que o aluno fumava e a quantidade de cigarros consumidos por dia, verificou-se que quanto maior o tempo de tabagismo, maior a quantidade de cigarros fumados por dia, sendo esta associação estatisticamente significativa $(\mathrm{p}<0,05)$.

Figura 1 - Prevalência de tabagismo nos cursos do Unisalesiano-Lins/SP.

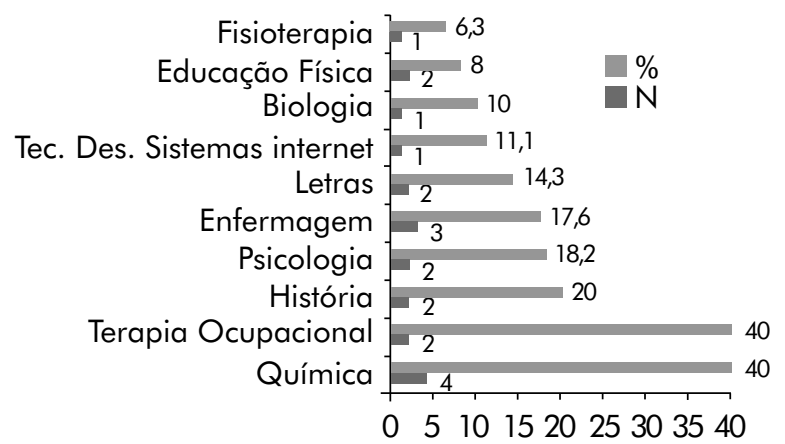

Considerando os hábitos e atitudes tabágicas, foram observadas diferenças estatisticamente significativas $(\mathrm{p}<0,05)$ entre os alunos fumantes regulares $(40,0 \%)$ e os ocasionais $(60,0 \%)$ para as questóes relacionadas ao fumar mesmo estando doente. Já em relação aos malefícios à saúde secundários ao uso de cigarros, não houve diferenças significativas $(\mathrm{p}>0,05)$ entre os fumantes regulares $(53,3 \%)$ e ocasionais $(60,0 \%)$. O mesmo se aplica ao aconselhamento de profissionais da saúde na cessação do tabagismo: fumantes regulares $(73,3 \%)$ e fumantes ocasionais $(80,0 \%)$.

Com relação aos sintomas decorrentes do hábito tabágico, 85,0\% (17) dos fumantes apresentam algum tipo de sintoma como: falta de ar, "chiado no peito", tosse seca ou gripe/resfriados frequentes, sendo a média de tempo de fumo entre estes de 6,3 anos. Entre todos os fumantes, $30 \%$ apresentam ou já apresentaram algum tipo de doença respiratória.

Dos motivos que levaram os pesquisados a começar a fumar, a influência dos amigos foi o mais citado, com 40,0\% das respostas. Em seguida, 30,0 $\%$ iniciaram nesta prática por vontade própria, e com 10,0 \% cada por modismo, influência dos pais e decepçóes (Figura 2).
Cerca de 55,0\% dos alunos fumantes fazem uso de bebidas alcoólicas e apenas 35,0\% deles praticam atividade física. Residem com os pais $60,0 \%$, e com outros universitários, $25,0 \%$.

Figura 2 - Motivos que desencadearam o hábito tabágico.

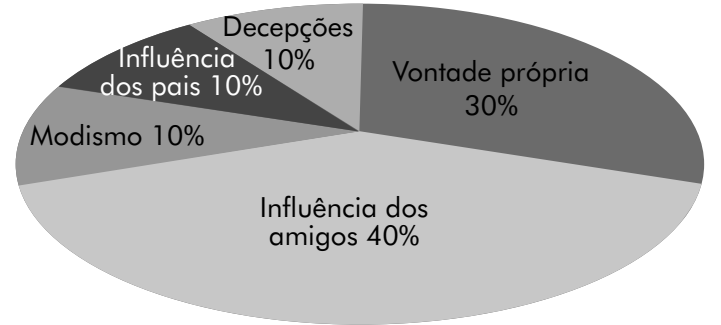

Dos alunos que se declararam ex-fumantes, todos responderam ter conseguido deixar de fumar por vontade própria.

A maioria dos alunos que se declararam não fumantes afirmou conviver com fumantes, o que representou $57,1 \%$ da amostra, sendo predominante a convivência com fumantes na universidade, com $44,3 \%$ das respostas dadas (Tabela III).

Tabela III - Distribuição dos alunos não fumantes de acordo com a convivência com fumantes.

\begin{tabular}{lll}
\hline Convivência com fumantes & $\mathrm{n}$ & $\%$ \\
\hline Universidade & 39 & 44,3 \\
Domicílio & 27 & 30,7 \\
Trabalho & 22 & 25,0 \\
Não convivem com fumantes & 66 & 42,9 \\
\hline
\end{tabular}

\section{Discussão}

Náo há outro fator de risco descrito na literatura capaz de causar taxas táo elevadas de morbimortalidade direta ou indiretamente como o tabagismo [12]. Por isso, este estudo propôs descrever o perfil e hábitos do consumo tabágico entre os estudantes de todos os cursos do Unisalesiano-Lins/SP.

Há cerca de um bilhão de homens fumantes no mundo, sendo que $50 \%$ deles estão em países em desenvolvimento. As tendências das prevalências de fumo no sexo masculino mostram uma mínima e lenta reduçáo, tanto nos países desenvolvidos como nos em desenvolvimento. No sexo feminino, ao contrário, encontram-se prevalências em elevação, principalmente em países em desenvolvimento [1].

Em nosso estudo, 67,6\% dos entrevistados foram do sexo feminino, e houve significância esta- 
tística para a prevalência de fumantes deste gênero $(80,0 \%)$, confirmando esta tendência mundial.

Em âmbito mundial, podem ser encontrados diversos estudos de prevalência tabágica, demonstrando características diferenciadas das encontradas no Brasil. Mesmo frente à tendência de crescimento do tabagismo entre universitários em âmbito internacional, no Brasil podemos observar o oposto, através de estudos que avaliaram a tendência tabágica entre os alunos das principais universidades brasileiras, que apontam para uma reduçáo do tabagismo entre os universitários nas últimas décadas [12]. Resultado semelhante foi observado em nosso estudo, em que a prevalência de tabagismo entre os universitários do Unisalesiano-Lins/SP foi de 11,0\%.

Um fator que merece destaque em estudos de prevalência de tabagismo é o tipo de tabaco mais utilizado em determinada população de fumantes. Alguns autores [8], ao estudarem as preferências de universitários norte-americanos pelo tipo de tabaco, detectaram que mais da metade dos alunos tabagistas utilizava cigarro industrializado. Segundo outros autores, este tipo de tabaco é o mais consumido entre os universitários, seguido por cachimbo e charuto [7]. Entre os alunos do Unisalesiano-Lins/SP, todos aqueles que se declararam fumantes utilizavam cigarros industrializados.

Esta preferência de consumo tabágico é preocupante e representa a realidade atual entre os jovens. Além disso, vale ressaltar que a idade de experimentação e início do hábito tabágico precoce, ou seja, antes dos vinte anos de idade, está comumente associada ao período de transição do indivíduo, do nível de ensino médio para o superior, sendo que muitos jovens podem apresentar o primeiro contato com o cigarro quando estáo ingressando na universidade. Além disso, em outro estudo foi relatado que mais de $50 \%$ dos estudantes tabagistas regulares tendiam a aumentar o consumo tabágico após a admissão ao ambiente universitário [10].

O primeiro contato com o cigarro para a maioria dos universitários ocorreu antes de 17 anos [13] e o tabagismo durante a adolescência é um preditor para o hábito na idade adulta [14]. Isso permite inferir que o consumo tabagístico seja predominante após os 25 anos. Uma grande parcela dos estudantes do Unisalesiano-Lins/SP (80,0\%), iniciou o hábito tabágico antes dos 20 anos.

Um ponto que merece destaque é o consumo de bebidas alcoólicas, pois, além de causar inúmeras doenças, quando do seu uso combinado ao tabaco, somam-se os efeitos nocivos deste último, além do alcoolismo ser fator dificultador para a interrupção do tabagismo [9]. Isto indica que quem bebe fuma mais, o que está de acordo com nossa pesquisa, haja vista que 55,0\% associam o uso do tabaco à ingestáo de álcool.

Ao se avaliar a opiniáo dos estudantes fumantes do Unisalesiano-Lins/SP em relação à capacidade e pretensão de cessar o hábito tabágico, observou-se que $90,0 \%$ dos universitários tabagistas supunham ser capazes de interromper o hábito, subestimando, assim, sua dependência ao cigarro.

Embora 70\% dos fumantes manifestem desejo de parar de fumar, somente $5 \%$ conseguem fazê-lo sem auxílio de profissionais da saúde. Além disso, há escassez no número e no treinamento de profissionais capacitados para abordar o paciente tabagista e empreender medidas de intervenção. Ademais, o número de serviços de apoio para abandono do fumo é insuficiente, assim como a distribuição gratuita de medicamentos utilizados na cessação do tabagismo na rede pública [2].

Os alunos da área de ciências biológicas devem merecer um enfoque diferenciado em relaçáo ao uso de álcool e de outras drogas, pois, futuramente, são eles que levaráo as noçóes básicas de saúde à comunidade. Assim, é importante conhecer o padrão de consumo, as atitudes e o conhecimento em relação às drogas entre esses alunos [15].

As prevalências de tabagismo entre estudantes de cursos da área de saúde são inferiores aos resultados encontrados entre alunos de outros cursos superiores [9]. Alguns autores verificaram menores prevalências de estudantes fumantes entre os procedentes das faculdades de ciências da saúde dentre todos os estudantes da Universidade de Jordânia [7]. Entretanto, na presente pesquisa, observou-se que a prevalência de tabagismo nos cursos dessa área alcançou o terceiro e quarto lugares (Psicologia e Enfermagem, respectivamente).

$\mathrm{O}$ aconselhamento profissional sobre os malefícios decorrentes do tabaco faz-se importante instrumento na luta contra o tabagismo. De acordo com nosso estudo, $25,0 \%$ dos fumantes nunca receberam orientaçóes sobre o hábito tabágico, o que demonstra uma lacuna nas políticas de prevenção nas instituiçóes de ensino superior. A alta prevalência de fumantes interessados em deixar de fumar sugere que, neste grupo, açóes para cessação do tabagismo seriam eficazes e possivelmente trariam efeitos positivos [13]. 
Além disso, outra característica importante encontrada foi o fato de grande parcela de universitários não tabagistas conviverem de forma involuntária com fumantes, principalmente na universidade, gerando repercussóes proporcionalmente maléficas na saúde.

\section{Conclusão}

Os estudantes universitários tabagistas caracterizam-se por conhecer os malefícios ocasionados pelo tabaco e por subestimar sua dependência ao mesmo. Estes estudantes estão vivendo na primeira fase da história natural do tabagismo e apostando com a sorte de não ter doenças futuras. Motivá-los a parar de fumar seria a melhor abordagem para esse grupo.

Assim, os resultados obtidos nesta pesquisa podem ajudar a elaborar estratégias para a cessação do hábito e náo iniciação no mesmo nessa população. Algumas medidas que podem ser adotadas são: detecçáo precoce do hábito, fornecimento de informação científica, programas de professores/ tutores (que seriam instruídos e treinados para detectar problemas dessa ordem), e como recomenda a American College of Health Association e a American Cancer Society, proibição da venda, publicidade e distribuiçáo de amostras gratuitas de produtos derivados do tabaco nos campi universitários, além de vetar o uso do tabaco em todos os locais da instituição. Ainda, maior carga horária para as disciplinas que abordam o uso de álcool e drogas lícitas e ilícitas é uma medida interessante.

Ao identificar os grupos mais expostos, podemos direcionar uma política de conscientização e prevenção para esta população, podendo ser mais bem sucedida do que uma ação que aborde igualmente a todos.

\section{Referências}

1. World Health Organization. Male smoking: tobacco free initiative. Geneva: WHO; 2003.

2. Azevedo RCS, Higa CMH, Assumpção ISAM, Fernandes RF, Boscolo MM, Frazatto CRG. Atenção aos tabagistas pela capacitaçáo de profissionais da rede pública. Rev Saúde Pública 2008;42(2):353-5.

3. Malcon MC, Menezes AMB, Chatkin M. Prevalência e fatores de risco para tabagismo em adolescentes. Rev Saúde Pública 2003;37(1):1-7.

4. Andrade APA, Bernardo ACC, Viegas CAA, Ferreira DBL, Gomes TC, Sales MR. Prevalência e características do tabagismo em jovens da Universidade de Brasília. J Bras Pneumol 2006;32(1):23-8.

5. Brasil. Instituto Nacional do Câncer. Ação global para o controle do tabaco. Primeiro Tratado Internacional de Saúde Pública. Brasília: Ministério da Saúde; 2002.

6. Moskal PD, Dziuban CD, West GB. Examining the use of tobacco on college campuses. J Am Coll Health 1999;47(6):260-5.

7. Haddad LG, Malak MZ. Smoking habits and attitudes towards smoking among university students in Jordan. Int J Nurs Stud 2002;39(8):793-802.

8. Rigotti NA, Lee JE, Wechsler H. US college students use of tobacco products: results of a national survey. JAMA 2000;284(6):699-705.

9. Baska T, Straka S, Mad'ar R. Smoking habits in university students in Slovakia. Cent Eur J Public Health 2000;8(4):245-8.

10. Halperin AC, Rigotti NA. US public universities' compliance with recommended tobacco-control policies. J Am Coll Health 2003;51(5):181-8.

11. Oliveira EFT, Grácio MCC. Análise a respeito do tamanho de amostras aleatórias simples: uma aplicação na área de Ciência da Informação. Revista Ciência da Informação 2005;6(3).

12. Menezes A, Horta BL, Oliveira AL. Attributed risk to smoking for lung cancer, laryngeal cancer and esophageal cancer. Rev Saúde Publica 2002;36:129-34.

13. Rodrigues ESR, Cheik NC, Mayer AF. Nível de atividade física e tabagismo em universitários. Rev Saúde Pública 2008;42(4):672-8.

14. Escobedo LG, Marcus SE, Holtzman D, Giovino GA. Sports participation, age at smoking initiation, and the risk of smoking among US High School students. JAMA 1993;269(11):1391-5.

15. Silva LVER, Malbergier A, Stempliuk VA, Andrade AG. Fatores associados ao consumo de álcool e drogas entre estudantes universitários. Rev Saúde Pública 2006;40(2):280-8. 